\title{
Stable RNA markers for identification of blood and saliva stains revealed from whole genome expression analysis of time-wise degraded samples
}

\author{
Dmitry Zubakov • Eline Hanekamp • \\ Mieke Kokshoorn • Wilfred van IJcken • \\ Manfred Kayser
}

Received: 3 January 2007 / Accepted: 16 May 2007 /Published online: 20 June 2007

(C) Springer-Verlag 2007

\begin{abstract}
Human body fluids such as blood and saliva represent the most common source of biological material found at a crime scene. Reliable tissue identification in forensic science can reveal significant insights into crime scene reconstruction and can thus contribute toward solving crimes. Limitations of existing presumptive tests for body fluid identification in forensics, which are usually based on chemoluminescence or protein analysis, are expected to be overcome by RNA-based methods, provided that stable RNA markers with tissue-specific expression patterns are available. To generate sets of stable RNA markers for reliable identification of blood and saliva stains we (1) performed whole-genome gene expression analyses on a series of timewise degraded blood and saliva stain samples using the
\end{abstract}

Electronic supplementary material The online version of this article (doi:10.1007/s00414-007-0182-6) contains supplementary material, which is available to authorized users.

D. Zubakov $\cdot$ M. Kokshoorn $\cdot$ M. Kayser $(\bowtie)$

Department of Forensic Molecular Biology,

Erasmus University Medical Center Rotterdam,

P.O. Box 2040, 3000 Rotterdam, The Netherlands

e-mail: m.kayser@erasmusmc.nl

E. Hanekamp

Department of Biology, Netherlands Forensic Institute,

P.O. Box 24044, 2490 Den Haag, The Netherlands

W. van IJcken

Erasmus Center for Biomics,

Erasmus University Medical Center Rotterdam,

P.O. Box 2040, 3000 Rotterdam, The Netherlands

Present address:

E. Hanekamp

Department of Pathology, Leiden University Medical Center,

P.O. Box 9600, 2300 Leiden, The Netherlands
Affymetrix U133 plus2 GeneChip, (2) consulted expression databases to obtain additional information on tissue specificity, and (3) confirmed expression patterns of the most promising candidate genes by quantitative real-time polymerase chain reaction including additional forensically relevant tissues such as semen and vaginal secretion. Overall, we identified nine stable mRNA markers for blood and five stable mRNA markers for saliva detection showing tissuespecific expression signals in stains aged up to 180 days of age, expectedly older. Although, all of the markers were able to differentiate blood/saliva from semen samples, none of them could differentiate vaginal secretion because of the complex nature of vaginal secretion and the biological similarity of buccal and vaginal mucosa. We propose the use of these 14 stable mRNA markers for identification of blood and saliva stains in future forensic practice.

Keywords Body fluid identification - Gene expression . Blood $\cdot$ Saliva $\cdot$ Biological traces $\cdot$ RNA markers

\section{Introduction}

Human body fluids such as blood and saliva are the most common sources of biological trace material found at a crime scene. Reliable tissue identification in forensic casework is important as it provides crucial insights into crime scene reconstruction and can thus contribute towards solving crimes. Blood stains are routinely tested in forensic practise using various methods including the tetrabase (4,4-bis (dimethylamino)diphenylmethane) test [1], the KastleMeyer phenolphthalein test, the tetramethylbenzidine test [2], the orthotolidine test [3], or the luminol (3-aminophthalhydrazide) chemoluminescence test [4], with the latter 
especially appropriate for detecting blood stains after cleaning attempts $[2,5]$. All these presumptive-thus indicative but not identifying - tests take advantage of the peroxidase-like activity of the heme unit of the hemoglobin molecule in human blood. Therefore, false-positive results can be caused by the presence of strong oxidants, such as chlorine-containing detergents or by true peroxidases (e.g., from plants) [6].

Saliva stains are usually detected in forensic practise via an enzymatic amylase test using Phadebas [7] or with a recently developed enzyme-linked immunosorbent assaybased method [8]. However, because of amylase degradation, the time window for the successful performance of such tests can be limited [9]. Furthermore, no amylase assay can distinguish between salivary amylase and amylases from other tissues (pancreatic, urinary, etc.); therefore, the tests for saliva identification are only presumptive (similar to existing blood identification tests).

On the other hand, methods for identification and quantification of mRNA are already well established, although mostly outside the forensic field. These methods make massive multiplex gene expression profiling possibleamong many other applications-for the discovery of tissue-specific mRNA markers. The major concern of using mRNA markers for forensic applications is their assumed high susceptibility to degradation. However, recent studies using a few selected genes demonstrated that it is possible to isolate total RNA of sufficient quality and quantity from biological stains that are several months or even years old [10-12]. It has also been suggested, although with limited evidence so far, that different types of mRNA seem to follow different rates of degradation [13]. It is assumed that the degradation process of mRNA is influenced by many external and internal factors, including structural peculiarities like the presence of AU-rich elements (ARE motifs), protein binding properties, and cellular localization [14, 15]. However, detailed knowledge on the molecular reasons for differences in RNA degradation between different types of RNAs as well as between mRNAs of different genes is currently lacking and further investigations are sorely needed.

Although a small number of mRNA markers has been tested for tissue identification in forensic science [16-19], no systematic study has yet been performed. In addition, the identification of candidate markers in previous studies was based on a mixed literature and database search, apparently without strict criteria of selection, considering only a limited number of genes and tissues, and not taking into account RNA degradation levels. Furthermore, expressed sequence tags databases, which were used previously, like the Cancer Genome Anatomy Project [18], are expected to provide heavily biased information on candidate genes because of the nonrandom character of representation of clone libraries.
To find stable mRNA markers for body fluid identification in forensic practice, we performed a systematic and comprehensive whole-genome gene expression analysis on time-wise degraded blood and saliva stains using the Affymetrix U133 plus2 GeneChip. This expression array contains $>54,000$ mRNA probe sets, which encompass most, if not all, known and predicted human genes. Tissuespecific expression patterns of the most promising candidate genes from the array analyses were further confirmed using the GNF SymAtlas expression database [20], which covers about 100 human tissues, and finally verified by quantitative real-time polymerase chain reaction (PCR) in blood and saliva as well as in other body fluids relevant for forensic casework, i.e., semen and vaginal secretion.

\section{Materials and methods}

\section{Sample collection}

Aliquots of $5 \mathrm{ml}$ of whole blood and saliva were collected from each of five healthy volunteers (four men and one woman) of western European genetic origin under informed consent before their inclusion in the study. Native blood was collected without anticoagulation treatment to avoid disturbing effects of anticoagulation reagents on gene expression. In each sample, 75 cotton swabs were immersed. Special care was taken to shorten the time between collection and swab absorption to avoid blood coagulation. After complete absorption of the fluids, swabs were left until dry on a bench top at room temperature. When dry, the swabs were stored in dust-free nonhumid conditions (but subjected to normal daylight) for different time intervals. Swabs were visually inspected and sorted out to ensure similar liquid content between individual swabs. After 0,1 , $3,7,14,21,57$, and 180 days, swabs were stored at $-80^{\circ} \mathrm{C}$ until RNA isolation. For the time interval 0 days, samples were frozen immediately after drying. Semen and vaginal secretion samples were collected from one male and one female individual absorbed with cotton swabs and dried overnight before RNA isolation.

\section{RNA isolation}

RNA was isolated using the Qiagen RNeasy kit (Qiagen Benelux B.V.) according to the manufacturer's instructions with minor modifications. These included cutting up the cotton swab into $1 \times 1-\mathrm{mm}$ pieces and soaking them in RLT buffer for $1 \mathrm{~h}$ at $4^{\circ} \mathrm{C}$ before the extraction. Trial experiments to lengthen this incubation time up to $24 \mathrm{~h}$ did not reveal any improvement in respect to RNA quantity and quality (data not shown). 
Microarray hybridization and gene expression data analysis

Before hybridization to Affymetrix U133 plus2.0 GeneChip arrays (Affymetrix, Santa Clara, CA), RNA isolated from blood and saliva stains was amplified using the Ambion MEGAscript T7 two-cycle amplification kit (Applied Biosystems, The Netherlands). Amplification, labeling, hybridization, washing, and scanning were performed by the microarray core facility of the Erasmus MC Center for Biomics according to Affymetrix specifications. Background subtraction and probe signal summarization were calculated according to the robust multiarray analysis algorithm [21] using the R Bioconductor software [22]; the resulting $\log 2$ signal values were back-transformed to linear scale. Presence/absence calls for individual probe sets were calculated with the mas5calls function of the Bioconductor mas package. Because the constant global mean assumption does not hold true for arrays hybridized to differentially degraded RNA samples, the normalization of the signal intensities between samples was performed using the nonhuman control genes present on Affymetrix arrays (spiked-in probes). Normalization factors for each array were inferred from the average signal intensities of bioB, bioC, bioD, and Cre control probe sets. Analysis of differential gene expression was performed using the significance analysis of microarrays (SAM) algorithm [23] implemented in the TM4 software [24]. In the saliva dataset, we selected only genes with signal intensities above 50 (which is below the usually applied background threshold in expression array experiments) that had a signal intensity below 50 in the blood dataset. The selection of blood-targeted genes was done in a similar manner but with different criteria, the lower intensity limit in blood was set to 1,000 to reasonably restrict the number of candidates.

\section{Real-time PCR}

First strand cDNAs were synthesized with SuperScript ${ }^{\circledR}$ III RTS First-Strand cDNA Synthesis Kit (Invitrogen BV, The Netherlands) using total RNA as a template. The primers were designed with Primer3 software [25] so that forward and reverse primers were complementary to different exons of the respective genes and most closely located to the 3'end of the corresponding RefSeq cDNA (Electronic Supplementary Material Table S1). Real-time PCR reactions with the SuperScript ${ }^{\circledR}$ III Platinum ${ }^{\circledR}$ SYBR $^{\circledR}$ Green One-Step qPCR Kit (Invitrogen BV) were performed on an ABI 7300 PCR machine (Applied Biosystems, The Netherlands) using the following parameters: initial denaturation at $94^{\circ} \mathrm{C}$ for $10 \mathrm{~min}$, followed by 45 cycles of denaturation at $94^{\circ} \mathrm{C}$ for $15 \mathrm{~s}$, and a final annealing/elongation at $60^{\circ} \mathrm{C}$ for $30 \mathrm{~s}$. Melting profiling and agarose gel electrophoresis were used to confirm the specificity of the primers and the absence of DNA contamination. Quantification of the amplified cDNA yield in comparative blood and saliva PCRs was done by the standard curve method. PCR experiments with semen and vaginal secretion were quantified using delta $\mathrm{C}_{\mathrm{t}}\left(\mathrm{dC}_{\mathrm{t}}\right)$ method. In both cases, GAPDH gene was used as an endogenous control to normalize the amplification signal between the samples from different tissues and individuals. Time points were compared to each other without normalization: Assuming the temporal degradation of all RNA molecules, no internal control gene could be used, and the only proper way to normalize RT-PCR signals was to use the same amount of template in each reaction. We found that this requirement holds true for our experiments because the GAPDH expression variability between different samples from the same tissue was relatively low ( $\mathrm{CV}<25 \%$, data not shown), which is probably because of approximately the same amount of blood or saliva absorbed with cotton swabs during material collection.

\section{Results and discussion}

\section{Microarray expression data}

As expected, hybridization signals demonstrated high variability between individuals; however, the most striking differences were observed between the different tissues. Signal intensities in blood samples were on average about five times higher than in saliva (174.2 \pm 1.9 in blood samples vs $26.9 \pm 0.7$ in saliva; Wilcoxon test rank sum $p<$ $0.001)$. In addition, at the time-point zero, the number of the probe sets called as present according to the Affymetrix algorithm was, on average, more than three times higher in blood than in saliva $(30.2 \% \pm 0.9$ vs $9.3 \% \pm 0.6 ; t$ test $p<$ 0.001). The SAM test with stringent parameters (false discovery rate was set to $0 \%$ ) showed that, both in blood and saliva experiments, no genes demonstrated significant expression differences in a time range of 0-57 days of stain storage. Only few genes ( 37 and 10 significantly differential genes for saliva and blood, respectively) appeared to be differentially expressed at 180 days in comparison to other time points. This suggests that in dried blood and saliva, mRNA molecules remain relatively stable for a long period. Recent studies of Heinrich et al. [26] also revealed poor correlation between RNA degradation and postmortem time intervals.

Selection of tissue-specific markers

The initial selection of tissue-specific genes was performed using the normalized signal intensities of microarray hybridizations averaged across the five biological replicates 
at the zero experimental time point. About 500 apparent saliva-specific and 1,000 apparent blood-specific candidate genes were selected. Further refinement of tissue-specific gene sets was achieved by probing the selected candidates against the GNF SymAtlas tissue database [20] after excluding all cell lines from the database retaining only tissues and organs for the analysis. Genes were selected only if they were highly and exclusively expressed in the target tissue(s) based on the GNF SymAtlas database. For blood, target tissue in the database was defined as whole blood; while for saliva, the target tissues were salivary gland, tongue, trachea, and tonsils. The selection criteria were as follows: high expression (signal intensity $>1,000$ ) in target tissue and low expression (signal intensity $<200$ ) in nontarget tissues. Using these criteria and combining data from expression array experiments as well as GNF SymAtlas database verification, we identified six salivatargeted genes and 15 blood-targeted genes that were highly expressed only in target tissues (or respective organs) but not, or nearly not, in the nontarget tissues (Electronic Supplementary Material Figure S1a, S1b, S1c).

\section{RT-PCR confirmation of tissue-specific markers}

To confirm the microarray results, real-time PCR experiments were designed for the 21 best candidate markers selected from array hybridizations and database searches and performed using RNA extracted from aged blood and saliva stains, also providing a method suitable for forensic applications. In agreement with the array results, all 21 markers analyzed showed good amplification in the target tissue but no or only marginally detectable amplification in the nontarget tissue (Fig. 1a-c). Among the candidate markers, only the PPL gene that was targeted for saliva demonstrated significant expression overlap with blood and therefore was excluded from further experiments. Our results demonstrate that, irrespective of the stain storage time, sufficient RT-PCR amplification was observed in all samples, even in the samples from the longest storage time tested (180 days), indicating marker stability over long periods of sample storage time. The only exception was the CCR2 gene for which no amplification was detected in the blood stains stored for 180 days and was therefore excluded from further analyses. A plausible explanation for this peculiarity could be the location of the PCR-amplified region, which is more than $1 \mathrm{~kb}$ distant from the $3^{\prime}$ end of the mRNA because of the very long untranslated region of CCR2. Apparently, the degree of degradation of the CCR2 mRNA after 180 days of sample storage was too high to allow its efficient reverse transcription using the oligo(dT) method that targets the 3 'end of the molecule. This observation highlights the necessity to design PCR primers for the most 3'-proximal part of the mRNA molecule for successful amplification of cDNA fragments in degraded samples.

Expression of the candidate markers in other body fluids

For additional confirmation of the tissue-specificity, we tested by RT-PCR the expression patterns of our candidate RNA markers in other body fluids that might be observed in a forensic case, i.e., vaginal secretion and semen. According to the GNF SymAtlas database, all our markers targeted for blood and saliva are not expressed in testis nor in uterus tissues. In agreement, our dedicated RT-PCR experiments revealed that two of the saliva-targeted mRNA markers (SPRR3 and SPRR1A) show no detectable expression in semen (after 50 RT-PCR cycles), and the remaining three (KRT4, KRT6A, and KRT13) show vast overexpression in saliva compared to semen $\left(\operatorname{ddC}_{t}>15\right.$, Fig. 2a), keeping with the assumption of high saliva specificity of the five proposed mRNA markers. SPRR1A and SPRR3 genes both encode cornified envelope precursor proteins and are predominantly expressed in oral and esophageal epithelia, where they are strictly linked to keratinocyte terminal differentiation [27]. Keratins 4, 6A, and 13 are known as one of the major structural proteins of oral mucosa [28, 29].

For the 14 blood-targeted genes, we observed no detectable amplification in semen for nine genes (CASP1, AMICA1, C1QR1, ALOX5AP, AQP9, C5R1, NCF2, MNDA, ARHGAP26), keeping with the assumption of high blood specificity of the respective mRNA markers. These genes encode the proteins with important functions in different types of blood cells. They are known to be highly or even specifically expressed in peripheral leukocytes (AQP9, NCF2, CASP1, C5R1, C1QR1, ALOX5AP [30-35]) and myelocytes or hematopoietic cells (MNDA, ARHGAP26, AMICA1 [36-38]). However, five genes demonstrated only slightly differential or even comparable expression in blood and semen (CD36, CCR1, PF4, BIN2, and ALOX5), not expected given the information provided by the GNF database, and were therefore excluded from the final list of blood-specific markers. Thus, our microarraybased genome-wide approach to find tissue-specific mRNA markers identified the genes that are functionally relevant for the target tissues.

Furthermore, and not surprisingly, RT-PCR of all salivaand blood-targeted markers in samples from vaginal secretion revealed gene expression at a level comparable to that in blood and saliva samples (Fig. 2b). The natural occurrence of blood cells in vaginal secretion most likely explains the expression of our blood-targeted markers in vaginal secretion, whereas the high biochemical and histological similarity of oral and vaginal epithelia [40] makes the similarity of gene expression patterns between 
Fig. 1 a, b RT-PCR results for blood-targeted genes in blood and saliva stains. c RT-PCR

results for saliva-targeted genes in saliva and blood stains. Genes were selected based on expression microarray results and GNF SymAtlas database. Expression values for each time point were averaged across three male and three female RNA samples; no gender-specific expression differences were detected $(t$ test $p<$ $0.05)$. $B$ indicates blood; $S$ indicates saliva; samples were processed after complete drying of blood and saliva at $0,21,57$, and 180 days, respectively a

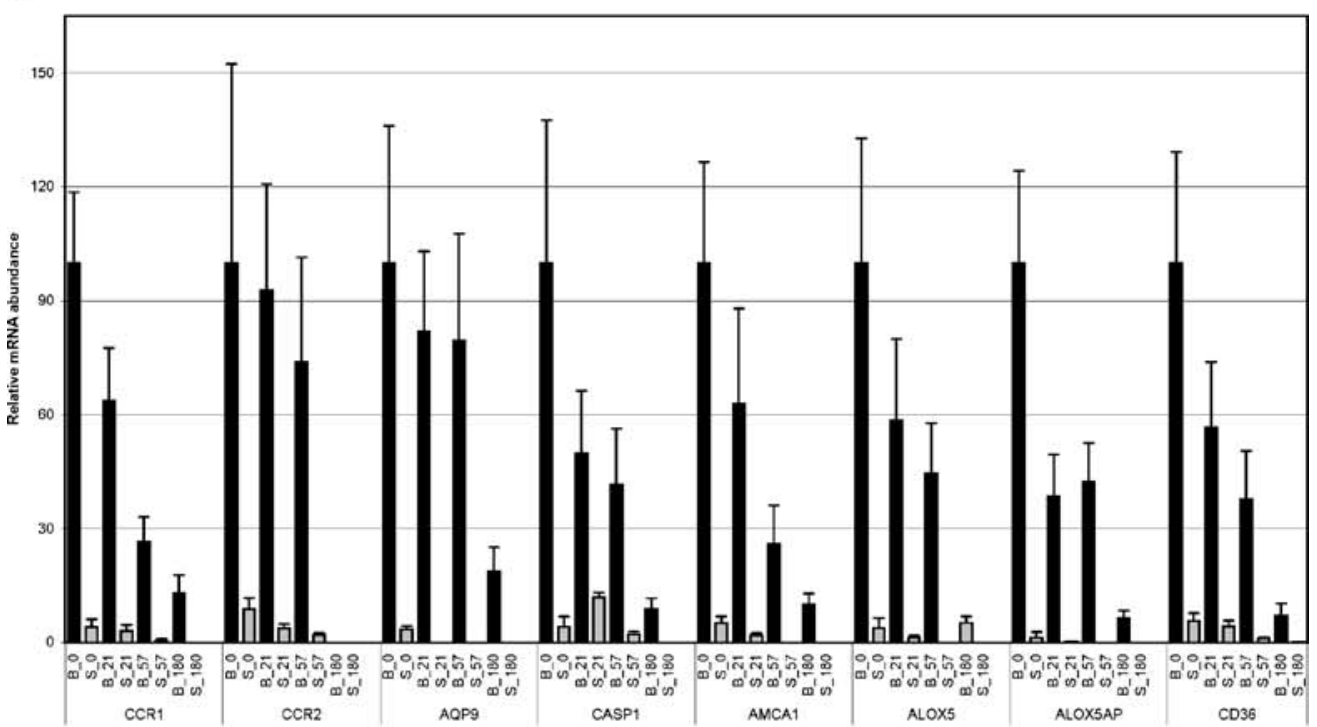

b

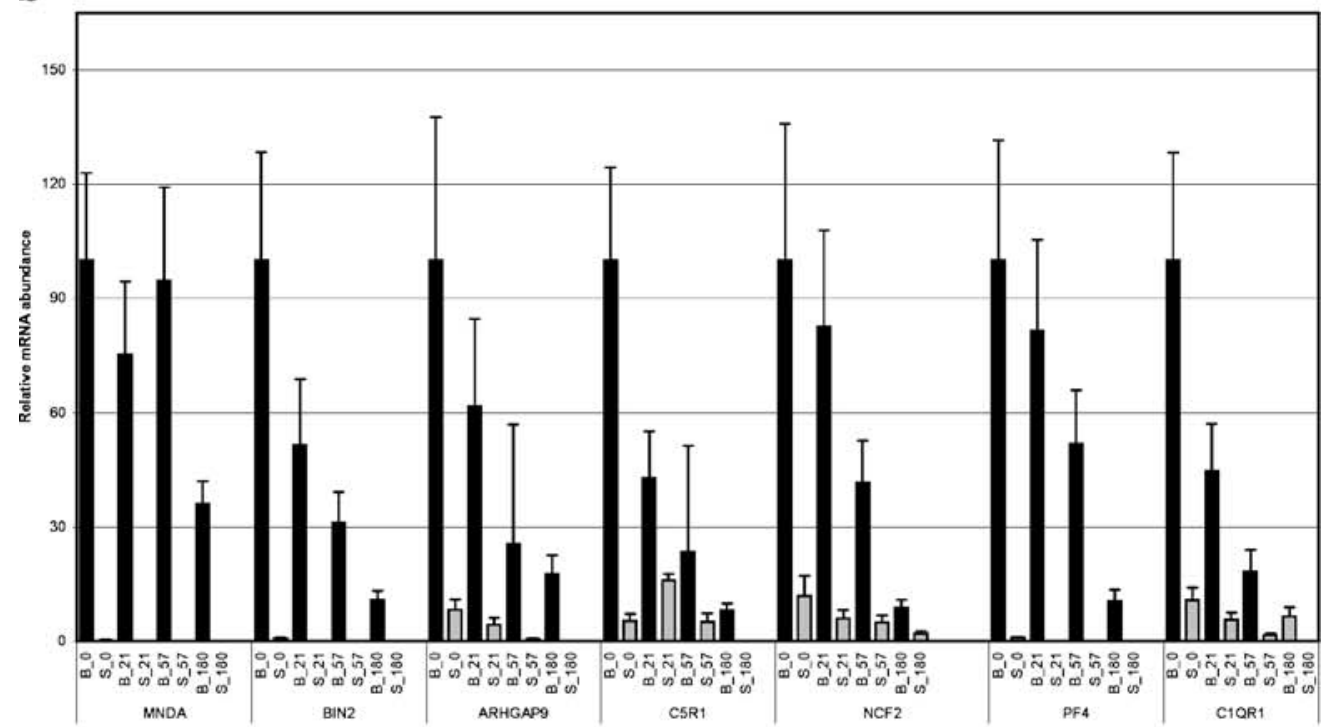

C

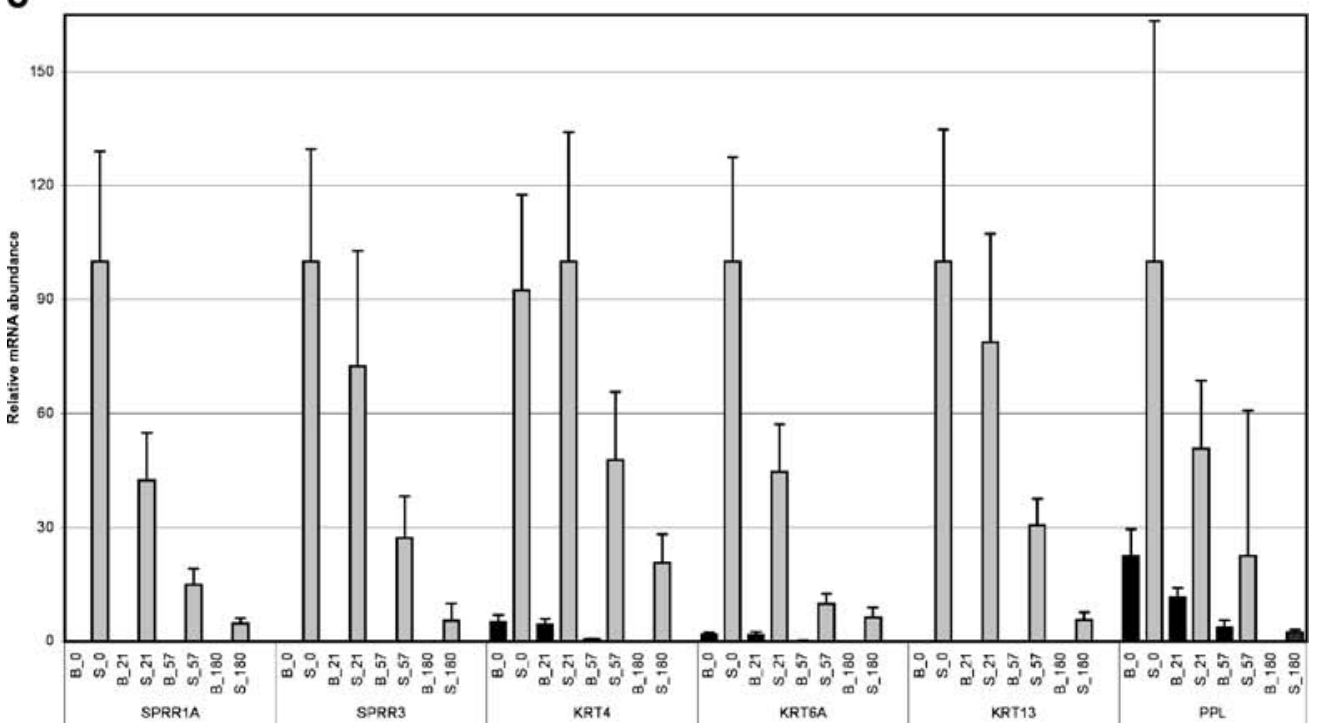


both tissues plausible. It should be pointed out that mRNA markers previously claimed to be useful for the identification of vaginal secretion such as HBD-1 [18] and MUC4 $[18,19]$ are known to be abundant also in oral epithelial cells and the salivary transcriptome [41-43]. Furthermore, Nussbaumer et al. [19] ruled out the potential to differentiate saliva and vaginal secretion using solely MUC4. Our results, together with previous findings, suggest that establishing mRNA markers expressed exclusively in vaginal secretion could be a challenging if not impossible task.

Comparison with previously suggested mRNA markers

Interestingly, tissue-specific genes, as identified here, do not overlap with the ones previously suggested for blood and saliva stain identification [18, 19]. This could be explained by the experimental setup and the systematic (but not ad hoc) approach of this study, namely, the degraded biological material analysed and the Affymetrix microarray platform applied. In contrast to previous studies, we restricted our marker ascertainment to those genes, which retained structural mRNA integrity during the stain dry-out process as well as the subsequent long-term storage of 180 days. This allows future application of detection of these markers in forensic stains of unknown age, at least up to an age of 6 months, but expectedly longer. Furthermore, our saliva-specific candidate genes were derived from mouth and pharynx epithelial cells, unlike the previously suggested STATH and HTN3 genes that are expressed in the salivary gland [18]. Secreted mRNAs that are abundant in fresh saliva are more prone to fast degradation by extracellular RNAses [39]; they are therefore not expected to be present in dried stains, explaining why they were not detected by the relatively low-sensitive microarray hybridization method used in this study. The SPTB and PBGD genes, previously proposed as blood-specific markers [18],
Fig. 2 a RT-PCR for saliva and blood-targeted genes in semen stains. b RT-PCR for saliva and blood targeted in vaginal secretion stains. Delta $\mathrm{C}_{\mathrm{t}}\left(\mathrm{dC}_{\mathrm{t}}\right)$ values were calculated as follows: $\mathrm{dC}_{\mathrm{t}}=\mathrm{C}_{\mathrm{t}}$ (candidate gene) $-\mathrm{C}_{\mathrm{t}}$ (endogenous control, GAPDH gene). Low $\mathrm{dC}_{\mathrm{t}}$ values correspond to high expression level of the specific mRNA. Gray bars correspond to the samples from target tissues for selected genes (either blood or saliva); black bars correspond to samples from nontarget tissues (either vaginal secretion or semen). Dotted bars represent the cases were amplification was not detected after 50 cycles, in this case, the expression values were arbitrary set to $C_{t}$ value of 25 (plot maximum)

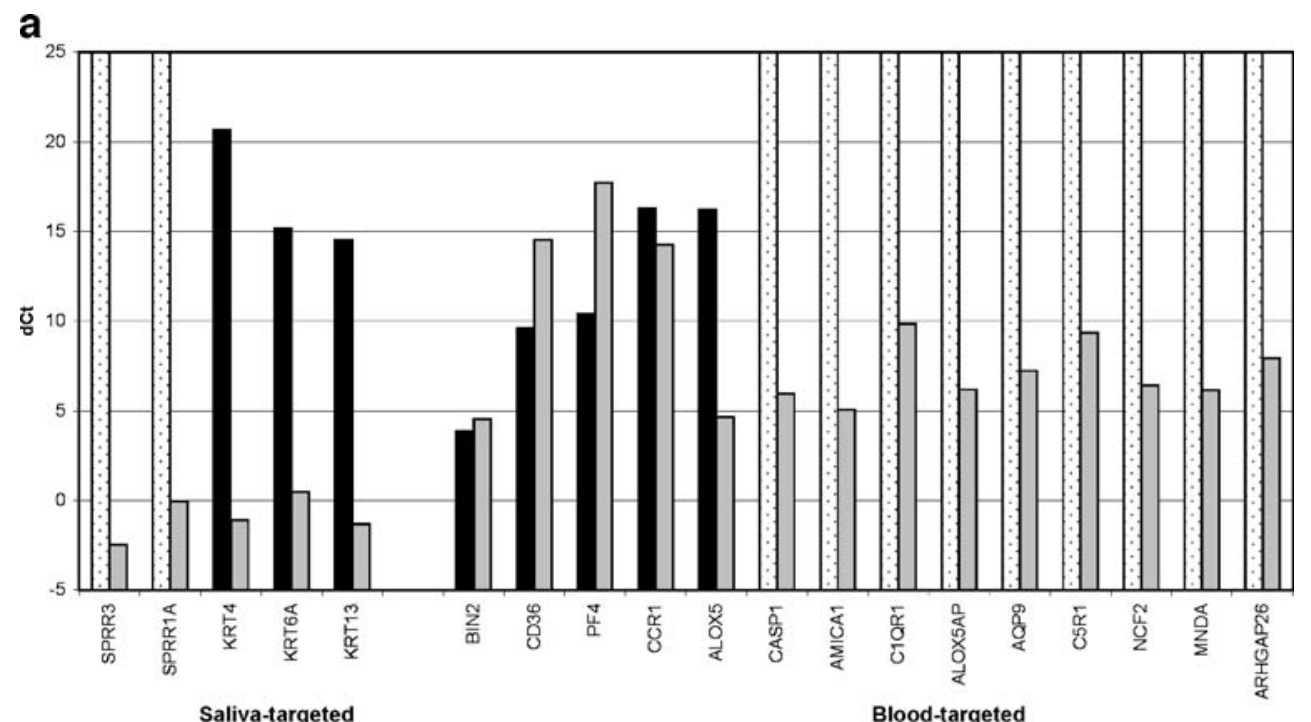

b

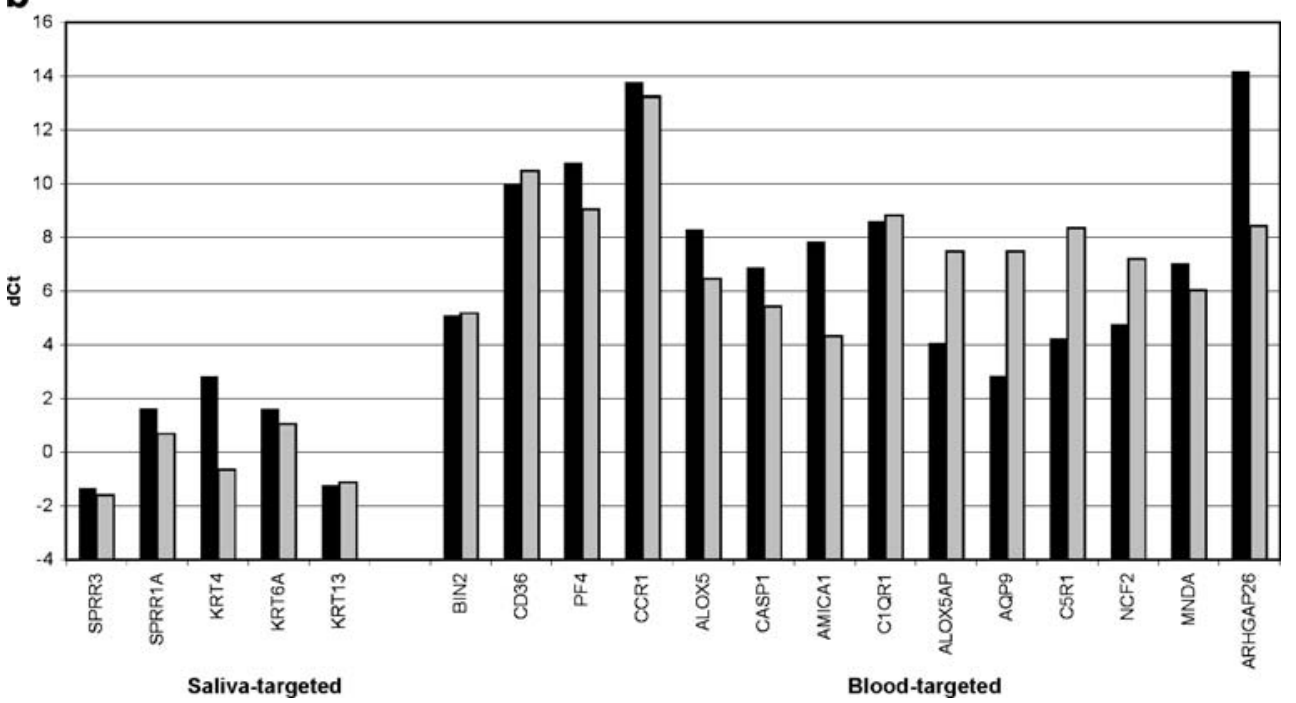


do not demonstrate any overexpression relative to other tissues in whole blood according to the GNF SymAtlas database (data not shown).

\section{Conclusions}

In summary, whole-genome expression analysis in timewise degraded samples from blood and saliva stains in combination with RT-PCR verification of various forensically relevant body fluids has resulted in the identification of stable tissue-specific mRNA markers from five genes for saliva (SPRR3, SPRR1A, KRT4, KRT6A, and KRT13) and nine genes for whole blood (CASP1, AMICA1, C1QR1, ALOX5AP, AQP9, C5R1, NCF2, MNDA, and ARHGAP26). For the first time, mRNA markers were ascertained considering almost the entire human transcriptome and based on experimental data of genome-wide gene expression as well as considering the degradation stability of mRNAs. We could demonstrate that the candidate genes identified here provide informative mRNA markers for blood and saliva identification for stains up to 180 days of age. We would like to propose their application in forensic case work (with the potential practical limitation of coamplification in vaginal secret) for stains of at least 6 months of age. However, we expect that the proposed mRNA markers will successfully identify older blood and saliva stains (respective experiments are currently in progress). Finally, we would like to remark that tissue identification in forensics should be performed in a reciprocal way; so that a tissue is identified because of the presence of markers specific for the relevant tissue together with the absence of markers specific for all other tissues in question. Clearly, more research should be dedicated towards finding the most suitable markers for tissue identification in forensics.

Acknowledgments We are grateful to all volunteers who provided tissue samples for this study. We thank Bianca de Graaf as well as Miriam Goedbloed and Silke Brauer for assistance in sample collections and preparations, and Nienke van Doorn for help in RNA extraction. Additional colleagues at the Erasmus MC Center for Biomics are acknowledged for microarray hybridization experiments. This study was supported by The Netherlands Forensic Institute, the Erasmus University Medical Center, and by additional funds from the Fonds Schiedam Vlaardingen to support forensic molecular biology at Erasmus MC. This study received additional support from the Translational Medicine Program of Affymetrix and Erasmus MC.

\section{References}

1. Lomholt B, Keiding N (1977) Tetrabase, an alternative to benzidine and orthotolidine for detection of haemoglobin in urine. Lancet 1:608-609
2. Garner DD, Cano KM, Peimer RS, Yeshion TE (1976) An evaluation of tetramethylbenzidine as a presumptive test for blood. J Forensic Sci 21:816-821

3. Kohn J, O'Kelly T (1955) An ortho-tolidine method for the detection of occult blood in faeces. J Clin Pathol 8:249-251

4. Weber K (1966) The use of chemiluminescence of luminol in forensic medicine and toxicology. I. Identification of blood stains. Dtsch Z Ges Gerichtl Med 57(3):410-423

5. Creamer JI, Quickenden TI, Crichton LB, Robertson P, Ruhayel RA (2005) Attempted cleaning of bloodstains and its effect on the forensic luminol test. Luminescence 20(6):411-413

6. Kent EJ, Elliot DA, Miskelly GM (2003) Inhibition of bleachinduced luminol chemiluminescence. J Forensic Sci 48(1):64-67

7. Foot CH, Wiener K (1979) Phadebas amylase test kits. Clin Chem 25(5):818

8. Quarino L, Dang Q, Hartmann J, Moynihan N (2005) An ELISA method for the identification of salivary amylase. J Forensic Sci 50(4):873-876

9. Keating SM, Higgs DF (1994) The detection of amylase on swabs from sexual assault cases. J Forensic Sci Soc 34(2):89-93

10. Ferri G, Bini C, Ceccardi S, Pelotti S (2004) Successful identification of two years old menstrual bloodstain by using MMP-11 shorter amplicons. J Forensic Sci 49(6):1387

11. Karlsson H, Guthenberg C, von Dobeln U, Kristenssson K (2003) Extraction of RNA from dried blood on filter papers after longterm storage. Clin Chem 49(6 Pt 1):979-981

12. Anderson S, Howard B, Hobbs GR, Bishop CP (2004) A method for determining the age of a bloodstain. Forensic Sci Int 3:22-24

13. Meyer S, Temme C, Wahle E (2004) Messenger RNA turnover in eukaryotes: pathways and enzymes. Crit Rev Biochem Mol Biol 39(4): 197-216

14. Brewer G (2002) Messenger RNA decay during aging and development. Age Res Rev 1:607-625

15. Ross J (1996) Control of messenger RNA stability in higher eukaryotes. Trends Genet 24(5):171-175

16. Bauer M, Patzelt D (2002) Evaluation of mRNA markers for the identification of menstrual blood. J Forensic Sci 47(6):1278-1282

17. Juusola J, Ballantyne J (2003) Messenger RNA profiling: a prototype method to supplant conventional methods for body fluid identification. Forensic Sci Int 135:85-96

18. Juusola J, Ballantyne J (2005) Multiplex mRNA profiling for the identification of body fluids. Forensic Sci Int 152:1-12

19. Nussbaumer C, Gharehbaghi-Schnell G, Korschineck I (2006) Messenger RNA profiling: a novel method for body fluid identification by Real-Time PCR. Forensic Sci Int 157(2-3):181-186

20. Su AI, Cooke MP, Ching KA et al (2002) Large-scale analysis of the human and mouse transcriptomes. Proc Natl Acad Sci USA 99 (7):4465-4470

21. Irizarry RA, Hobbs B, Collin F, Beazer-Barclay YD, Antonellis KJ, Scherf U, Speed TP (2003) Exploration, normalization, and summaries of high density oligonucleotide array probe level data. Biostatistics 4:249-264

22. Gentleman RC, Carey VJ, Bates DM et al (2004) Bioconductor: open software development for computational biology and bioinformatics. Genome Biol 5(10):R80

23. Tusher VG, Tibshirani R, Chu G (2001) Significance analysis of microarrays applied to the ionizing radiation response. Proc Natl Acad Sci USA 98(9):5116-5121

24. Saeed AI, Sharov V, White J et al (2003) TM4: a free, open-source system for microarray data management and analysis. Biotechniques 34:374-378

25. Rozen S, Skaletsky H (2000) Primer3 on the WWW for general users and for biologist programmers. Methods Mol Biol 132:365-386

26. Heinrich M, Matt K, Lutz-Bonengel S, Schmidt U (2007) Successful RNA extraction from various human postmortem tissues. Int J Legal Med 121(2):136-142 
27. Gibbs S, Fijneman R, Wiegant J, van Kessel AG, van De Putte P, Backendorf C (1993) Molecular characterization and evolution of the SPRR family of keratinocyte differentiation markers encoding small proline-rich proteins. Genomics 1993 16(3):630-637

28. Guo JH, Maltha JC, He SG, Krapels IP, Spauwen PH, SteegersTheunissen RP, Von den Hoff JW (2006) Cytokeratin expression in palatal and marginal mucosa of cleft palate patients. Arch Oral Biol 51(7):573-580

29. Wong P, Colucci-Guyon E, Takahashi K, Gu C, Babinet C, Coulombe PA (2000) Introducing a null mutation in the mouse K6alpha and K6beta genes reveals their essential structural role in the oral mucosa. Cell Biol 150(4):921-928

30. Ishibashi K, Kuwahara M, Gu Y, Tanaka Y, Marumo F, Sasaki S (1998) Cloning and functional expression of a new aquaporin (AQP9) abundantly expressed in the peripheral leukocytes permeable to water and urea, but not to glycerol. Biochem Biophys Res Commun 244(1):268-274

31. Gauss KA, Bunger PL, Crawford MA et al (2006) Variants of the 5 '-untranslated region of human NCF2: expression and translational efficiency. Gene 366(1):169-179

32. Westphal E, Herzberg M, Neumann I, Beibei L, Pilowski C, Li C, Werdan K, Loppnow H (2006) Neutrophils process interleukin1beta and interleukin-18 precursors in a caspase-1-like fashionprocessing is inhibited by human vascular smooth muscle cells. Eur Cytokine Netw 17(1):19-28

33. Kastl SP, Speidl WS, Kaun C et al (2006) The complement component $\mathrm{C} 5 \mathrm{a}$ induces the expression of plasminogen activator inhibitor-1 in human macrophages via NF-kappaB activation. J Thromb Haemost 4(8):1790-1797

34. Nepomuceno RR, Tenner AJ (1998) C1qRP, the C1q receptor that enhances phagocytosis, is detected specifically in human cells of myeloid lineage, endothelial cells, and platelets. J Immunol 160 (4):1929-1935

35. Helgadottir A, Manolescu A, Thorleifsson G et al (2004) The gene encoding 5-lipoxygenase activating protein confers risk of myocardial infarction and stroke. Nat Genet 36(3):233-239

36. Xie J, Briggs JA, Briggs RC (1998) Human hematopoietic cell specific nuclear protein MNDA interacts with the multifunctional transcription factor YY1 and stimulates YY1 DNA binding. J Cell Biochem 70(4):489-506

37. Borkhardt A, Bojesen S, Haas OA et al (2000) The human GRAF gene is fused to MLL in a unique $t(5 ; 11)(q 31 ; q 23)$ and both alleles are disrupted in three cases of myelodysplastic syndrome/acute myeloid leukemia with a deletion 5q. Proc Natl Acad Sci USA 97:9168-9173

38. Moog-Lutz C, Cave-Riant F, Guibal FC et al (2003) JAML, a novel protein with characteristics of a junctional adhesion molecule, is induced during differentiation of myeloid leukemia cells. Blood 102(9):3371-3378

39. Thompson IO, van der Bijl P, van Wyk CW, van Eyk AD (2001) A comparative light-microscopic, electron-microscopic and chemical study of human vaginal and buccal epithelium. Arch Oral Biol 46(12):1091-1008

40. Park NJ, Li Y, Yu T, Brinkman BMN, Wong DT (2006) Characterization of RNA in saliva. Clin Chem 52:988-994

41. Schenkels LC, Veerman EC, Nieuw Amerongen AV (1995) Biochemical composition of human saliva in relation to other mucosal fluids. Crit Rev Oral Biol Med 6(2):161-175

42. Liu B, Lague JR, Nunes DP et al (2002) Expression of membraneassociated mucins MUC1 and MUC4 in major human salivary glands. J Histochem Cytochem 50(6):811-820

43. Abiko Y, Nishimura M, Kaku T (2003) Defensins in saliva and the salivary glands. Med Electron Microsc 36(4):247-252 